\title{
Self-Focusing of High-Power Laser Beam Through Cold Uniform Magnetized Plasma
}

\author{
Sudarshan Kumar Chakravarti* \\ University Department of Physics, T.M.B. University, Bhagalpur, India \\ *Corresponding author: sudarshanchakravarti5@gmail.com
}

\begin{abstract}
In present paper the determination of the spot size of an ultra-short laser beam in uniform magnetized plasma with a dominant cold plasma has been studied. The liner dispersion relation of the laser beam propagating in Magnetized plasma have been found. Magnetic field is set up and source dependent expansion method is applied to determining the spot size of the intense laser beam with gaussian profile. The transverse magnetization of plasma and its impact on the self-focusing property of the dense laser governing to reduction in critical power necessary to self-focusing beam is shown.
\end{abstract}

Keywords: Self-Focusing, Magnetized plasma, Ponderomotive force, Laser fusion.

\section{Introduction}

When Ultra-Short laser pulse of high intensity passes through the plasma, a nonlinear interaction between plasma and Laser gives a very important result. It is highly useful for the laser fusion, laser wake-field acceleration etc. The wide application of ultrafast laser pulse in various application and is very interesting laser plasma interaction in nonlinear Optics [1]-[3].

The interaction of intense laser pulse with magnetized plasma is very fascinating area of study. It is observed that, intense magnetic field produces in laser plasma interaction [4]. This field affects the penetration of ultra- short laser pulse in plasma because Canonical momentum for magnetized plasma interaction is not properly conserved as similar as in case of unmagnetized Plasma [5]. It is applicable in fast ignition scheme in inertial confinement fusion [6]. Where Quasi stable self- generated magnetic field may remain in the under dense Corona region near the critical surface of the ignition laser pulses. Modulation instability and the wave excitation are some useful method which are recently observed for intense ultrashort laser pulse interacting when penetrating through magnetized plasma [7], [8]. When ultrasonic laser pulse penetrating deep in magnetized plasma and ponderomotive force on electron, the plasma electron motion is modified due to magnetic field and gives a change in dispersion of the laser beam and due to magnetic field nonlinear current density is also affected [9]-[11].

In the present paper we have investigated the effect of the magnetic field over the self-focusing of highly intense laser pulse penetrating in a cold dense and homogeneous plasma. Since magnetic field is transvers to dielectric vector, the direction of penetration of the radiation forward the field. It is very interesting to investigate the transverse magnetic fields are produced in the laser plasma interaction and it is applicable in many useful events like modulation of the propagation of laser pulse in magnetized plasma. The paper is structured as follow,

In section 2, linear dispersion relation and produced nonlinear magnetic field due to transverse magnetic plasma is observed and in section 3 a wave equation in nonlinear form for a laser penetrating in magnetic plasma is derived. In Section 4 solution of envelop equation for laser spot is determined and required critical power to self-focusing of the laser beam in magnetized plasma is discussed. Section 5 presents the conclusion.

\section{Formulation}

When a linear polarized laser pulse propagated through a uniform plasma contained in a uniform external magnetic field $\mathrm{b}=\mathrm{b} \hat{\mathrm{e}}_{\mathrm{x}}$. The electric field vector of the radiation propagating along Z-direction is represented as,

$$
\mathrm{E}=\hat{\mathrm{e}}_{x} E(r, z, t) \cos \left(k_{o} z-\omega_{o} t\right)
$$

Where $\mathrm{E}$ is amplitude of the radiation field and $\omega_{\mathrm{o}}$ and $\mathrm{k}_{\mathrm{o}}$ are the frequency and wave number respectively. The wave equation is represented as,

$$
\left(\nabla^{2}-\frac{1}{c^{2}} \frac{\partial^{2}}{\partial t^{2}}\right) \mathrm{E}=\frac{4 \pi}{c^{2}} \frac{\partial j}{\partial t}
$$

The plasma current density $\mathrm{J}=-\mathrm{ne} v$ where is the velocity of the electron of plasma and $n$ is the electron density of the plasma. Relation between electromagnetic field and plasma electron in form of Lorentz force equation, represented by,

$$
\begin{aligned}
& \frac{d(\gamma v)}{d t}=-\frac{e E}{m}-\frac{e}{m c} v x(B+b) \\
& \text { and } \frac{\partial n}{\partial t}+\nabla \cdot(n v)=0
\end{aligned}
$$

Which governs the continuity equation $Y$ is the relativistic factor and $\mathrm{B}$ is the magnetic vector produced by the laser beam in plasma considering the Plasma in cold. In this case, it is assumed that plasma electron is at rest when external magnetic 
field is neglected. All equations for velocity is along $\mathrm{X}$ and $\mathrm{Z}$ direction applying perturbation technique is represented by,

$$
\begin{aligned}
& \frac{\partial v_{\chi}^{(1)}}{\partial t}=-\frac{e}{m} E_{x}+v_{z}^{(1)} \omega_{c} \\
& \frac{\partial v_{z}^{(1)}}{\partial t}=v_{x}^{(1)} \omega_{c}
\end{aligned}
$$

which is cyclotron frequency of the plasma electron.

Solving equation (4) and equation (5) by determining with respect to time $t$ is given by,

$$
\begin{aligned}
& \frac{\partial^{2} v_{x}^{(1)}}{\partial t^{2}}+\omega_{c}^{2} v_{x}^{(1)}=-\frac{e}{m} \omega_{o} E \sin \left(k_{o} z-\omega_{o} t\right) \\
& \frac{\partial^{2} v_{z}^{(1)}}{\partial t^{2}}+\omega_{c}^{2} v_{z}^{(1)}=\frac{e}{m} \omega_{c} E \cos \left(k_{o} z-\omega_{o} t\right)
\end{aligned}
$$

Solution of the equation (6) and (7) is given as,

$$
\begin{aligned}
& v_{x}^{(1)}=\frac{c a \omega_{o}^{2}}{\left(\omega_{o}^{2}-\omega_{c}^{2}\right)} \sin \left(k_{o} z-\omega_{o} t\right) \text { here } \mathrm{a}=\frac{e E}{m c \omega_{O}} \\
& v_{x}^{(1)}=\frac{-\omega_{o} \omega_{c} c a}{\left(\omega_{o}^{2}-\omega_{c}^{2}\right)} \cos \left(k_{o} z-\omega_{o} t\right)
\end{aligned}
$$

is normalized radiation field amplitude. Transverse quiver velocity grows because of the magnetic field and result gives generation of longitudinal velocity due to ponderomotive force acting on the plasma electrons. This governs relativistic mass of the plasma electrons and it modified the refractive index of the medium.

The similar method is used to obtain the second order electronic velocity. the second order equation can be easily solved by the first order velocity.

$\frac{\partial^{2} v_{x}^{(2)}}{\partial t^{2}}+\omega_{c}^{2} v_{x}^{(2)}=-\frac{c^{2} k_{o} a^{2} \omega_{c} \omega_{o}^{2}\left(\omega_{o}^{2}-4 \omega_{c}^{o}\right)}{2\left(\omega_{o}^{2}-\omega_{c}^{2}\right)^{2}} \mathrm{x} \sin 2\left(k_{o} z-\omega_{o} t\right)$

$\frac{\partial^{2} v_{Z}^{(2)}}{\partial t^{2}}+\omega_{c}^{2} v_{z}^{(2)}=\frac{c^{2} k_{o} a^{2} \omega_{o}\left(2 \omega_{o}^{4}-4 \omega_{o}^{2} \omega_{c}^{2}-\omega_{c}^{4}\right)}{2\left(\omega_{o}^{2}-\omega_{c}^{2}\right)^{2}} \times \cos 2\left(k_{o} z-\omega_{o} t\right)$

In uniform magnetic field $\mathrm{X}$ component of the velocity grows but when magnetic field is removed its value reduces to zero. Due to radiation field and magnetic field $\mathrm{Z}$ component of velocity remains same. In the same way the third order $x$ component of the velocity of electron when harmonics is neglected represented by,

$$
\begin{gathered}
\frac{\partial^{2} v_{x}^{(3)}}{\partial t^{2}}+\omega_{c}^{2} v_{x}^{(3)}=c a^{2}\left[\frac{c^{2} \omega_{o}^{2} \omega_{c}^{2} k_{o}^{2}\left(5 \omega_{o}^{4}-11 \omega_{c}^{2} \omega_{o}^{2}-6 \omega_{c}^{4}\right)}{4\left(\omega_{o}^{2}-\omega_{c}^{2}\right)^{3}\left(4 \omega_{o}^{2}-\omega_{c}^{2}\right)}+\right. \\
\left.\frac{\omega_{o}^{4}\left(3 \omega_{o}^{4}+2 \omega_{c}^{2} \omega_{o}^{2}+3 \omega_{c}^{4}\right)}{8\left(\omega_{o}^{2}-\omega_{c}^{2}\right)^{3}}\right] x \sin \left(k_{o} z-\omega_{o} t\right)
\end{gathered}
$$

With the help of first and second order quantities $\mathrm{x}$ component of third order velocity of the electron is given by,

$$
\begin{gathered}
v_{x}^{(3)}=-c a^{3}\left[\frac{c^{2} \omega_{o}^{2} \omega_{c}^{2} k_{o}^{2}\left(5 \omega_{o}^{4}-11 \omega_{c}^{2} \omega_{o}^{2}-6 \omega_{c}^{4}\right)}{4\left(\omega_{o}^{2}-\omega_{c}^{2}\right)^{4}\left(4 \omega_{o}^{2}-\omega_{c}^{2}\right)}+\right. \\
\left.\frac{\omega_{o}^{4}\left(3 \omega_{o}^{4}+2 \omega_{c}^{2} \omega_{o}^{2}+3 \omega_{c}^{4}\right)}{8\left(\omega_{o}^{2}-\omega_{c}^{2}\right)^{4}}\right] x \sin \left(k_{o} z-\omega_{o} t\right)
\end{gathered}
$$

But plasma laser interaction can be obtained introducing density perturbation equation in order to radiation field,

$$
\frac{\partial n^{(1)}}{\partial t}+n^{(0)}\left(\nabla \cdot \mathrm{V}^{(1)}\right)=0
$$

Here, ambient plasma electron density, equations show the longitudinal velocity produces first-order fluctuations in plasma density. Putting the value $\mathrm{v}$ in equation applying Transformers coulomb gauge $\left(\nabla_{\perp}\right.$.E=0) so, first order perturbation is given by,

$$
n^{(1)}=\frac{-n_{o} \omega_{c} c k_{o} a}{\left(\omega_{o}^{2}-\omega_{c}^{2}\right)} \cos \left(k_{o} z-\omega_{o} t\right)
$$

In presence of external magnetic-field the first order density perturbation come into play and it diminishes in absence of magnetic field.

The second order density response can be represented as,

$$
\frac{\partial n^{(2)}}{\partial t}+\nabla \cdot\left(n^{(0)} \mathrm{V}^{(2)}+n^{(1)} \mathrm{V}^{(1)}=0\right.
$$

Solving it we get,

$$
n^{(2)}=-\frac{c^{2} \omega_{o}^{2} n_{o} k_{o}^{2}\left(\omega_{o}^{2}-4 \omega_{c}^{2}\right.}{\left(\omega_{o}^{2}-\omega_{c}^{2}\right)^{2}\left(4 \omega_{o}^{2}-\omega_{c}^{2}\right)} \times \cos 2\left(k_{o} z-\omega_{o} t\right)
$$

The transverse current density can be obtained the perturbation velocities and densities as,

$J_{x=} J_{x}^{(1)}+J_{x}^{(3)}=-e\left(n^{(0)} v_{x}^{(1)}+n^{o} v_{x}^{(3)}+n^{(1)} v_{x}^{(2)}+n^{(2)} v_{x}^{(1)}\right.$

The $2^{\text {nd }}, 3^{\text {rd }}$ and $4^{\text {th }}$ term of the equation given nonlinear current density. external magnetic field affects the $2^{\text {nd }}$ and $4^{\text {th }}$ term because it changes realistic mass correction and density perturbation. $3^{\text {rd }}$ term of the equation comes due to lowest order longitudinal oscillation of Elections created by the magnetic field. Due to magnetic field, current density is responsible for modification of nonlinear refractive index. This governs the propagation characteristics of the laser beam in plasma. Putting the value of the $1^{\text {st }}, 2^{\text {nd }}$ and $3^{\text {rd }}$ order quantity in equation represents the current density and neglected all harmonics

$$
\begin{aligned}
& J_{x=}-e c a \mathrm{n}_{\mathrm{o}}\left[\frac{\omega_{o}^{2}}{\left(\omega_{o}^{2}-\omega_{c}^{2}\right)}-\mathrm{a}^{2}\left\{\frac{c^{2} \omega_{o}^{2} \omega_{c}^{2} k_{o}^{2}\left(5 \omega_{o}^{4}-11 \omega_{c}^{2} \omega_{o}^{2}-6 \omega_{c}^{4}\right)}{4\left(\omega_{o}^{2}-\omega_{c}^{2}\right)^{4}\left(4 \omega_{o}^{2}-\omega_{c}^{2}\right)}-\right.\right. \\
& \left.\left.\frac{c^{2} \omega_{o}^{2} k_{o}^{2}\left(2 \omega_{o}^{4}-9 \omega_{c}^{2} \omega_{o}^{2}-4 \omega_{c}^{4}\right)}{4\left(\omega_{o}^{2}-\omega_{c}^{2}\right)^{3}\left(4 \omega_{o}^{2}-\omega_{c}^{2}\right)}+\frac{\omega_{o}^{2}\left(3 \omega_{o}^{4}+2 \omega_{c}^{2} \omega_{o}^{2}-3 \omega_{c}^{4}\right)}{8\left(\omega_{o}^{2}-\omega_{c}^{2}\right)^{4}}\right\}\right] \mathrm{x} \\
& \sin \left(k_{o} Z-\omega_{o} t\right)
\end{aligned}
$$


Addition of liner current density in equation (2) governs the linear dispersion relation for a laser beam penetrating in the meantime plasma.

$$
\mathrm{c}^{2} k_{o}^{2}=\omega_{o}^{2}-\frac{\omega_{p}^{2} \omega_{o}^{2}}{\left(\omega_{o}^{2}-\omega_{c}^{2}\right)}
$$

For the value equation converted into the linear dispersion of the laser beam going through the plasma.

\section{Wave Dynamics}

Preparation of laser beam in nonlinear form in magnetized plasma can be derived by putting the current density in equation it can be represented as,

$$
\left(\nabla^{2}-\frac{1}{c^{2}} \frac{\partial^{2}}{\partial t^{2}}\right) \mathrm{a}=k_{p o}^{2}\left[\frac{\omega_{o}^{2}}{\left(\omega_{o}^{2}-\omega_{c}^{2}\right)}-a^{2} N\right] a
$$

Where,

$$
\begin{aligned}
& \mathrm{N}=\frac{c^{2} \omega_{o}^{2} \omega_{c}^{2} k_{o}^{2}\left(5 \omega_{o}^{4}-11 \omega_{c}^{2} \omega_{o}^{2}-6 \omega_{c}^{4}\right)}{4\left(\omega_{o}^{2}-\omega_{c}^{2}\right)^{4}\left(4 \omega_{o}^{2}-\omega_{c}^{2}\right)}-\frac{c^{2} \omega_{o}^{2} k_{o}^{2}\left(2 \omega_{o}^{4}-9 \omega_{c}^{2} \omega_{o}^{2}-4 \omega_{c}^{4}\right)}{4\left(\omega_{o}^{2}-\omega_{c}^{2}\right)^{3}\left(4 \omega_{o}^{2}-\omega_{c}^{2}\right)}+ \\
& \frac{\omega_{o}^{2}\left(3 \omega_{o}^{4}+2 \omega_{c}^{2} \omega_{o}^{2}-3 \omega_{c}^{4}\right)}{8\left(\omega_{o}^{2}-\omega_{c}^{2}\right)^{4}}
\end{aligned}
$$

In the above equation first term of the equation is the unperturbed linear current density for the laser beam penetrating into magnetized plasma and second term contains the nonlinear perturbation because relativistic effects. Coupling of radiation field following the magnetized field and density fluctuation.

Now the slowly varying function of $\mathrm{z}$ using paraxial ray approximation of the equation governs by,

$$
\left(\nabla_{\perp}^{2}+2 i k_{o} \frac{\partial}{\partial z}\right) a(r, Z)=k_{p 0}^{2}\left\{\frac{\omega_{o}^{2}}{\omega_{o}^{2}-\omega_{c}^{2}}-a^{2} N\right\} a(r, z)
$$

\section{Determination of Spot Size}

For getting the laser spot, we use SDE method. the laser field amplitude is represented as a source of Laguerre Gaussian source dependent mode. It can be represented as,

$$
\begin{aligned}
& \left.\mathrm{a}(\mathrm{r}, \mathrm{z})=\sum_{m} \hat{\mathrm{a}}_{m} L_{m}(\chi) \exp \left[-1-i \alpha_{s}\right) \chi / 2\right] \\
& \frac{\partial}{\partial z}\left(a_{s} r_{s}\right)=0 \\
& \frac{\partial^{2} r_{s}}{\partial z^{2}}=\frac{4\left(1+k_{o} r_{s}^{2} H\right)}{k_{o}^{2} r_{s}^{3}} \\
& \alpha_{2}=\frac{k_{o} r_{s}^{2}}{2 R_{c}}=k_{o} \frac{r_{s} \dot{\mathrm{r}}_{s}}{2} \text { and } \frac{\partial Q_{s}}{\partial t}=-\frac{2}{k_{o} r_{s}^{2}}-H-G
\end{aligned}
$$

It shows the total laser power dependence of z. So,

$$
\frac{\partial^{2} r_{S}}{\partial z^{2}}=\frac{4\left(1-\frac{k_{P O}^{2} a_{O}^{2} r_{O}^{2}}{8} N\right)}{3-}
$$
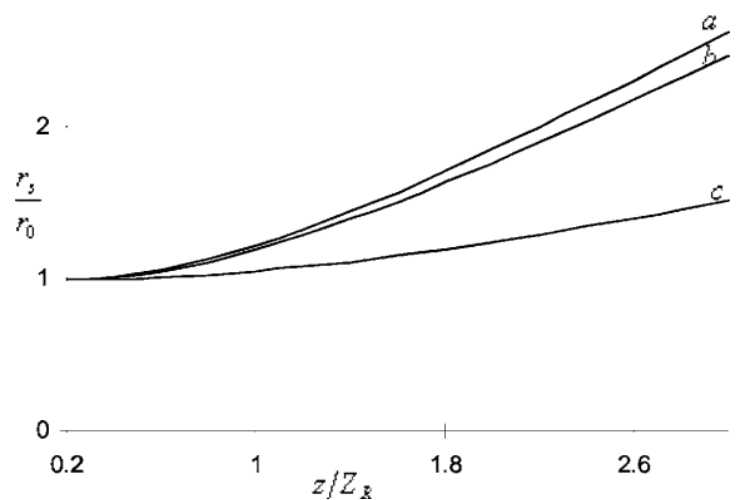

Fig. 1. Variation of $r_{s} / r_{0}$ with $z / Z_{R}$ for a unmagnetized plasma, $\mathrm{b} d_{0}=0.2$, and $\mathrm{c} d_{0}=0.4$ with $a_{0}=0.271,{ }_{0}=1.8810^{15} \mathrm{~s}^{-1}$, and ${ }_{p} / 0=0.1$

First term related to vacuum diffraction, second term is related to combined effect of the density perturbation relative mass correction and magnetic field which is useful for evaluation of the laser spot. Solution is given by,

$$
\begin{aligned}
& \frac{r_{S}^{2}}{r_{o}^{2}}=1+\left[1-\frac{P}{P_{C M}}\right] \frac{z^{2}}{Z_{R}^{2}} \\
& \frac{P}{P_{C M}}=\frac{k_{P O}^{2} a_{o}^{2} r_{o}^{2}}{8} N
\end{aligned}
$$

is the normalized power $\mathrm{d}$ and $\mathrm{Z}_{\mathrm{R}}$ represents the Raleigh length. It shows that when $\omega_{\mathrm{c}}=0 \mathrm{i}$.

i.e., magnetic field is not present, equation reduces to the spot size evolution. For laser penetration in the unmagnetized plasma. $\mathrm{P}$ shows critical power in magnetized plasma for nonlinear self-focusing and it is represented by,

$$
P_{C M}=\frac{2 \pi^{2} c^{2} m^{2}}{k_{p 0}^{2} \lambda^{2} e^{2} N}
$$

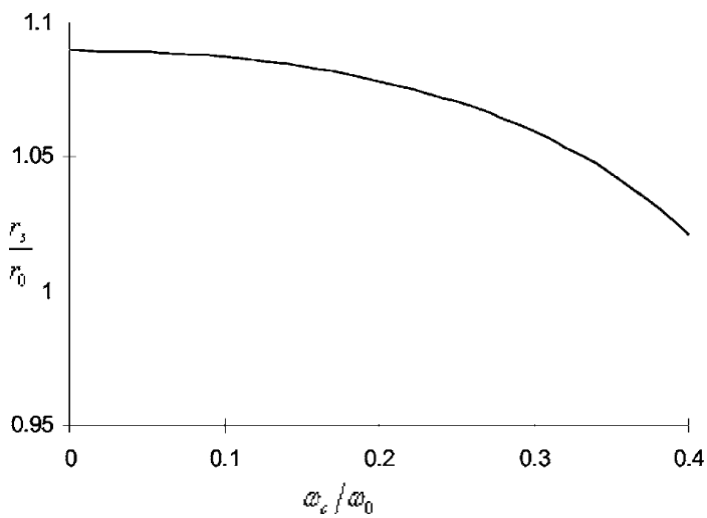

Fig. 2. Variation of $r_{s} / r_{0}$ with $d_{0}$ at $z / Z_{R}=0.3$ for $a_{0}=0.271,{ }_{0}=1.8810^{15} \mathrm{~s}^{-1}$, and ${ }_{p} / 0=0.1$ 


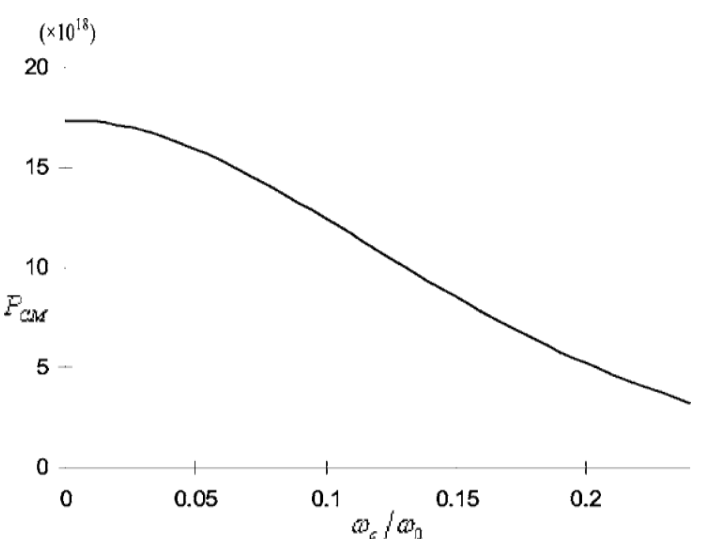

Fig. 3. Variation of $P_{C M}$ with $d_{0}$ for $a_{0}=0.271,{ }_{0}=1.8810^{15} \mathrm{~s}^{-1}$, and ${ }_{p} /{ }_{0}=0.1$

When magnetic field is not present it gets reduces to critical power needed for self-focusing of the laser beam.

In figure 1 , for frequency $\omega_{0}=1.88 \times 10^{15} \mathrm{~s}^{-2}$ and intensity $\mathrm{I}=10^{17} \mathrm{~W} / \mathrm{cm}^{2}$ is characterized against the normalized propagation distance $\frac{Z}{Z_{R}}$. for $\frac{\omega_{c}}{\omega_{o}}=0.4$ the laser spot grows to enhance because of magnetized Plasma.

The plot of $\frac{r_{s}}{r_{o}} \quad$ against $\frac{\omega_{c}}{\omega_{o}}$ is plotted to discuss the effect of increasing magnetized field to represent the laser spot. In fig. $\frac{Z}{Z_{R}}=0.3$ for $a_{\mathrm{o}}=0.271$.

When magnetic field is increased, sport size reduces. It showed that dependency of focusing of beam on value of magnetic field and it more focused when the magnetic field is enlarge. In fig. 3, the critical power needed for self-focusing of laser beam is drawn against $\frac{\omega_{c}}{\omega_{o}}$. It represents that the increase in magnetic field, it reflects the significant reduction in critical power needed for self-focusing of the laser beam when all the parameter is used same as in fig. 1.

\section{Conclusion}

In this work, the propagation of laser beam in varying magnetic plasma is discussed. The ponderomotive force acting on electrons of Plasma shows the change in relativistic mass and leads to the electron density perturbation and modification in the propagation of the laser beam. The present work reveals that transvers magnetization of plasma increases the selffocusing characteristics of the laser beam propagating in magnetized plasma and the increase in magnetic field represents a decrease in the laser spot size. The critical power needed to self-focusing of the beam in the magnetized plasma has been investigated. This work is very helpful in study the propagation of laser beam in magnetized plasma.

\section{References}

[1] Charls C "Plasma for Spacecraft population". Appl. Phys. (2009) 42:163001

[2] Fridman G, Fridman A, Gutsol A, 'Plasma Process Poiym, (2008) 5:507

[3] Hershhowitz N. "how Langmuir probs: plasma Diayaosties Vol-1 of plasma material Interactions.san Diego, CA: Acadmic press Inc (1989), pp. 111-117.

[4] W. B. Gombosi;, T.I. Roussev,I et al 2004, J.Geophy.Manchaster Res, 108,A01102.

[5] Reimann, K. U "Theory of the plasma sheath transition in $\mathrm{n}$ oblique magnetic field", 34:129-133, 1994.

[6] Harch R. "Numerical investigation of seath structure in presence of magnetic field" journal of phy.207:012030, 1-9, 2010.

[7] S. S. Esmaeily, and S. Jazavandi, "Ion dynamics in plasma seath under the effect of ExB and collisional force, 84:384-398, 2010.

[8] D. B. Montgomery, "Super conducting Magnets" IEEE spe.1, 106, 1964.

[9] T. G Ridgers, G Bell, C. P, Hirk, A.R, "Quantum Radiation Reaction in Laser Electron Beam Collising," Phy Rev. Lett 112(1);055001, 2014.

[10] G. Miley, "Laser Interaction and Related plasma phenomena," 4A, plenum press, 181, 1977.

[11] N. Sternberg and A. I. Smolyakov, "Resonant Transmission of Electromagnetic Waves in Multilayer Dense-Plasma Structures," in IEEE Transactions on Plasma Science, vol. 37, no. 7, pp. 1251-1260, July 2009. 